\title{
ESCAPE TIME FROM POTENTIAL WELLS OF STRONGLY NONLINEAR OSCILLATORS WITH SLOWLY VARYING PARAMETERS
}

\author{
JIANPING CAI, Y. P. LI, AND XIAOFENG WU \\ Received 29 July 2004 and in revised form 7 October 2004
}

The effect of negative damping to an oscillatory system is to force the amplitude to increase gradually and the motion will be out of the potential well of the oscillatory system eventually. In order to deduce the escape time from the potential well of quadratic or cubic nonlinear oscillator, the multiple scales method is firstly used to obtain the asymptotic solutions of strongly nonlinear oscillators with slowly varying parameters, and secondly the character of modulus of Jacobian elliptic function is applied to derive the equations governing the escape time. The approximate potential method, instead of Taylor series expansion, is used to approximate the potential of an oscillation system such that the asymptotic solution can be expressed in terms of Jacobian elliptic function. Numerical examples verify the efficiency of the present method.

\section{Introduction}

The effect of negative damping or external excitation to an oscillatory system may force the amplitude to increase gradually and the motion will eventually be out of the potential well of the oscillatory system. Escape from a potential well is a ubiquitous phenomenon in science. Examples are known in mechanics [16], chemistry [7], physics [2, 10], electronics [9], and so forth. Many efforts have been done to control a nonlinear dynamical system against escape from a potential well, which is often identified with system failure $[5,8,17]$. Bosley and Kevorkian derived an easily evaluated condition to predict the distance at which the electron escapes from the ponderomotive potential well [1]. An important parameter to characterize the dynamics of system is escape time. Coffey et al. obtained the escape time for rigid Brownian rotators [6]. Kevorkian and Li calculated the escape time as an application example of the Kuzmak-Luke method [12] and then $\mathrm{Li}$ generalized it to cubic nonlinear oscillator with slowly varying parameters [15]. Only the case of symmetric oscillation with a stationary oscillation center was studied there. This paper will develop the procedure in $[12,15]$ to drive the escape time from potential wells of quadratic and cubic nonlinear oscillators. Symmetric and asymmetric oscillations with stationary or varying oscillation centers are studied in detail. 
We begin with the following strongly nonlinear oscillator with slowly varying parameters:

$$
\frac{d^{2} y}{d t^{2}}+\varepsilon k(y, \tilde{t}) \frac{d y}{d t}+g(y, \tilde{t})=0
$$

where $\tilde{t}=\varepsilon t$ is the slow scale. Assume that functions $k$ and $g$ are arbitrary nonlinear functions of their arguments and (1.1) has periodic solutions when $\varepsilon=0$. Firstly, the multiple scales method is applied to obtain the asymptotic solutions of (1.1). Secondly, the character of modulus of Jacobian elliptic function is used to deduce the escape time from the potential well of quadratic or cubic nonlinear oscillator. Different signs of coefficients of quadratic and cubic polynomials will deduce different equations to determine the escape time. For a strongly nonlinear spring in Example 4.3, the approximate potential method, proposed by the authors in $[4,14]$, is applied to approximate the potential of oscillatory system such that the asymptotic solution can be expressed in terms of Jacobian elliptic function. Three examples are given to illustrate the main idea of this paper. Comparisons of asymptotic and numerical results are also made to show the efficiency of present method.

\section{Asymptotic solution of strongly nonlinear oscillator}

We assume that the solution of (1.1) can be developed in the form

$$
y(t, \varepsilon)=y_{0}\left(t^{+}, \tilde{t}\right)+\varepsilon y_{1}\left(t^{+}, \tilde{t}\right)+\varepsilon^{2} y_{2}\left(t^{+}, \tilde{t}\right)+\cdots,
$$

where $\tilde{t}=\varepsilon t$ is the slow scale. The fast scale $t^{+}$must be chosen such that the period is independent of $\tilde{t}$ when measured on the $t^{+}$scale (see [11, Section 3.6] for more details). Following Kuzmak [13], the fast scale $t^{+}$is defined as $d t^{+} / d t=\omega(\tilde{t})$ with an unknown $\omega(\tilde{t})$ to be determined by the periodicity of solution of (1.1). Substituting (2.1) into (1.1) and equating coefficients of like power of $\varepsilon$ yield the following equations:

$$
\begin{gathered}
\omega^{2}(\tilde{t}) \frac{\partial^{2} y_{0}}{\partial t^{+2}}+g\left(y_{0}, \tilde{t}\right)=0, \\
\omega^{2}(\tilde{t}) \frac{\partial^{2} y_{n}}{\partial t^{+2}}+g_{y}\left(y_{0}, \tilde{t}\right) y_{n}=F_{n}\left(y_{0}, y_{1}, \ldots, y_{n-1}, \tilde{t}\right),
\end{gathered}
$$

where $n=1,2, \ldots . F_{1}$ can be worked out in the form

$$
F_{1}=-2 \omega \frac{\partial^{2} y_{0}}{\partial t^{+} \partial \tilde{t}}-\frac{d \omega}{d t} \frac{\partial y_{0}}{\partial t^{+}}-\omega k\left(y_{0}, \tilde{t}\right) \frac{\partial y_{0}}{\partial t^{+}}
$$

Note that there is a periodic solution to the homogeneous equation (2.3) in the form

$$
y_{\mathrm{I}}=\frac{\partial y_{0}}{\partial \varphi},
$$


where $\varphi=t^{+}+\varphi_{0}, \varphi_{0}$ is constant and is determined by initial conditions [14]. The other solution linearly independent of $y_{\mathrm{I}}$ can be found by the reduction of order

$$
y_{\mathrm{II}}=y_{\mathrm{I}} \int_{0}^{\varphi} \frac{1}{y_{\mathrm{I}}^{2}} d \psi
$$

Unfortunately, the solution $y_{\mathrm{II}}$ is no longer periodic to general nonlinear system. Using variation of parameters, we obtain the general solution of the inhomogeneous equation (2.3) in the form

$$
\begin{aligned}
y_{n} & =C_{n}(\tilde{t}) y_{\mathrm{I}}+D_{n}(\tilde{t}) y_{\mathrm{II}}-\frac{y_{\mathrm{I}}}{\omega^{2}} \int_{0}^{\varphi} F_{n} y_{\mathrm{II}} d \psi+\frac{y_{\mathrm{II}}}{\omega^{2}} \int_{0}^{\varphi} F_{n} y_{\mathrm{I}} d \psi \\
& =y_{\mathrm{I}}\left[C_{n}(\tilde{t})+\int_{0}^{\varphi} \frac{d \psi}{y_{\mathrm{I}}^{2}}\left(D_{n}(\tilde{t})+\frac{1}{\omega^{2}} \int_{0}^{\psi} F_{n} y_{\mathrm{I}} d \gamma\right)\right],
\end{aligned}
$$

where coefficients $C_{n}$ and $D_{n}$ can be determined by the periodicity of higher-order solutions. To have $y_{n}$ periodic in $\varphi$, the inner integral and the outer integral in (2.7) must be periodic in $\psi$ and $\varphi$, respectively. We thus have, with the periodic normalized to be $T$,

$$
\begin{gathered}
\int_{0}^{T} F_{n} y_{\mathrm{I}} d \varphi=0, \\
\int_{0}^{T} \frac{d \varphi}{y_{\mathrm{I}}^{2}}\left(D_{n}(\tilde{t})+\frac{1}{\omega^{2}} \int_{0}^{\varphi} F_{n} y_{\mathrm{I}} d \psi\right)=0 .
\end{gathered}
$$

This paper just concerns applications of leading-order approximations. For more details of higher-order solution, readers can refer to [12] or [14].

Substituting (2.4) and (2.5) into (2.8) with $n=1$ yields

$$
\int_{0}^{T}\left(2 \omega f_{\varphi \tilde{t}} f_{\varphi}+\left(\frac{d \omega}{d \tilde{t}}+\omega k(f, \tilde{t})\right) f_{\varphi}^{2}\right) d \varphi=0 .
$$

This can be written as

$$
\frac{d}{d \tilde{t}}\left(\omega \int_{0}^{T} f_{\varphi}^{2} d \varphi\right)+\omega \int_{0}^{T} k(f, \tilde{t}) f_{\varphi}^{2} d \varphi=0
$$

Integrating (2.11) gives the following equation to determine $\omega(\tilde{t})$ :

$$
\omega(\tilde{t})=\frac{c}{\int_{0}^{T} f_{\varphi}^{2} d \varphi} \exp \left(\int_{0}^{\tilde{t}} \frac{\int_{0}^{T} k(f, \tau) f_{\varphi}^{2} d \varphi}{\int_{0}^{T} f_{\varphi}^{2} d \varphi} d \tau\right),
$$

where $c$ is an integral constant. If the damping $k$ depends on $y$, the calculation of $\omega(\tilde{t})$ will be rather involved. An approach of average damping is proposed in [14]. Instead of $k$, we use the leading term of its Taylor series expansion around $f=y_{r}$, the oscillatory center, that is, we assume

$$
k(y, \tilde{t})=k\left(y_{r}, \tilde{t}\right)+k_{y}\left(y_{r}, \tilde{t}\right)\left(y-y_{r}\right)+\frac{1}{2} k_{y y}\left(y_{r}, \tilde{t}\right)\left(y-y_{r}\right)^{2}+\cdots,
$$


where $y_{r}$ is the oscillatory center of system $(1.1)$ and is determined by $g\left(y_{r}, \tilde{t}\right)=0$. Because the system oscillates around the center $y_{r}$, the second term of (2.13) vanishes on average. Therefore, substitution of $k(y, \tilde{t}) \approx k\left(y_{r}, \tilde{t}\right)$ into (2.12) should give a good approximation for $\omega(\tilde{t})$. The result is

$$
\omega(\tilde{t})=\frac{c}{\int_{0}^{T} f_{\varphi}^{2} d \varphi} \exp \left(\int_{0}^{\tilde{t}} k\left(y_{r}, \tau\right) d \tau\right) .
$$

Numerical examples in Section 4 show that the results are quite satisfactory.

\section{Calculations of escape time from potential well}

3.1. Quadratic nonlinear oscillator. We now apply the results summarized in the previous section to the following quadratic nonlinear system:

$$
\frac{d^{2} y}{d t^{2}}+\varepsilon k(y, \tilde{t}) \frac{d y}{d t}+a(\tilde{t}) y+b(\tilde{t}) y^{2}=0
$$

Suppose that the solution of (3.1) can be developed in the form of asymptotic expression (2.1). The leading-order equation corresponding to (2.2) has the form

$$
\omega^{2}(\tilde{t}) \frac{\partial^{2} y_{0}}{\partial t^{+2}}+a(\tilde{t}) y_{0}+b(\tilde{t}) y_{0}^{2}=0
$$

Its energy integral is

$$
\frac{\omega^{2}(\tilde{t})}{2}\left(\frac{\partial y_{0}}{\partial t^{+}}\right)^{2}+V\left(y_{0}, a, b\right)=E_{0}(\tilde{t})
$$

where

$$
V\left(y_{0}, a, b\right)=\frac{1}{2} a(\tilde{t}) y_{0}^{2}+\frac{1}{3} b(\tilde{t}) y_{0}^{3}
$$

is the potential, and $E_{0}(\tilde{t})$ is the slowly varying energy of the system. It can be seen from (3.4) that the potential $V$ has a minimum at $y_{0}=0$ for the case of $a(\tilde{t})>0$. So (3.1) has periodic solutions around $y_{0}=0$ and the oscillatory center is at $y_{r}=0$. For the case of $a(\tilde{t})<0$, the potential $V$ has a minimum at $y_{0}=-a(\tilde{t}) / b(\tilde{t})$. Equation (3.1) has periodic solutions around $y_{0}=-a(\tilde{t}) / b(\tilde{t})$ and the oscillatory center $y_{r}=-a(\tilde{t}) / b(\tilde{t})$ is moving slowly with time.

3.1.1. Case of $a(\tilde{t})>0$. For this case, the solution of (3.2) can be expressed in terms of Jacobian elliptic cosine function [3]

$$
y_{0}=A_{0}(\tilde{t}) c n^{2}[K(v) \varphi, v(\tilde{t})]+B_{0}(\tilde{t})
$$


where $\varphi=t^{+}+\varphi_{0}$, and $K(v)$ is the complete elliptic integral of the first kind associated with the modulus $\sqrt{v}$. Substituting (3.5) into (3.2) yields

$$
\begin{gathered}
2 \omega^{2} K^{2} A_{0}(1-v)+a B_{0}+B_{0}^{2}+A_{0}\left[4 \omega^{2} K^{2}(2 v-1)+a+2 b B_{0}\right] c n^{2}(u, v) \\
+A_{0}\left(b A_{0}-6 \omega^{2} K^{2} v\right) c n^{4}(u, v)=0
\end{gathered}
$$

where $u=K(v) \varphi$. From (3.6), we obtain algebraic equations

$$
\begin{gathered}
2 \omega^{2} K^{2} A_{0}(1-v)+a B_{0}+B_{0}^{2}=0, \\
A_{0}\left[4 \omega^{2} K^{2}(2 v-1)+a+2 b B_{0}\right]=0, \\
A_{0}\left(b A_{0}-6 \omega^{2} K^{2} v\right)=0 .
\end{gathered}
$$

Then, we have

$$
\begin{aligned}
A_{0} & =\frac{3 a v}{2 b \sqrt{v^{2}-v+1}}, \\
B_{0} & =-\frac{a}{2 b}\left(\frac{2 v-1}{\sqrt{v^{2}-v+1}}+1\right), \\
\omega^{4} & =\frac{a^{2}}{16 K^{4}\left(v^{2}-v+1\right)} .
\end{aligned}
$$

Substituting (3.5) into (2.14), we get another form of $\omega(\tilde{t})$

$$
\omega^{5}=\frac{c b^{2}}{144 K^{5} v^{2} J(v)} \exp \left(-\int_{0}^{\tilde{t}} k(0, \tau) d \tau\right),
$$

where

$$
\begin{aligned}
J(v) & =\int_{0}^{K} s n^{2}(u, v) c n^{2}(u, v) d n^{2}(u, v) d u \\
& =\frac{1}{15 v^{2}}\left[(1-v)(v-2) K(v)+2\left(v^{2}-v+1\right) E(v)\right] .
\end{aligned}
$$

From (3.10) and (3.11), we have an equation for $v$

$$
\frac{v^{2} J(v)}{\left(v^{2}-v+1\right)^{5 / 4}}=\frac{2 c b^{2}}{9 a^{5 / 2}} \exp \left(-\int_{0}^{\tilde{t}} k(0, \tau) d \tau\right),
$$

where constant $c$ can be determined by initial values of the system.

It can be seen from (3.4) that the potential well is " $\sim$-shaped" or " $\sim$-shaped" when $a(\tilde{t})>0$. In this case, if there exists the effect of a negative damping in the system, the amplitude will be gradually increasing, and the motion will be forced out of the potential well and cease to be periodic. Obviously from (3.8), if $a / b$ is constant, the increasing amplitude implies an increasing modulus $v$. Once $v$ reaches $1, y_{0}$ is no longer a periodic function of $\varphi$. This can be used to determine the escape time from potential well. Denote 
the escape time as $T_{0}$, from (3.13) we have

$$
\frac{c b^{2}\left(T_{0}\right)}{a^{5 / 2}\left(T_{0}\right)} \exp \left(-\int_{0}^{\varepsilon T_{0}} k(0, \tau) d \tau\right)=\frac{3}{5} .
$$

Here, the fact that $J(v) \rightarrow 2 / 15$ as $v \rightarrow 1$ has been used.

3.1.2. Case of $a(\tilde{t})<0$ and $b(\tilde{t})>0$. For this case, the solution of (3.2) also can be expressed as (3.5) with

$$
\begin{gathered}
A_{0}=\frac{-3 a v}{2 b \sqrt{v^{2}-v+1}}, \\
B_{0}=-\frac{a}{2 b}\left(\frac{1-2 v}{\sqrt{v^{2}-v+1}}+1\right), \\
\omega^{4}=\frac{a^{2}}{16 K^{4}\left(v^{2}-v+1\right)} .
\end{gathered}
$$

Substituting (3.5) into (2.14), we get another form of $\omega(\tilde{t})$

$$
\omega^{5}=\frac{c b^{2}}{144 K^{5} v^{2} J(v)} \exp \left(-\int_{0}^{\tilde{t}} k\left(-\frac{a(\tau)}{b(\tau)}, \tau\right) d \tau\right) .
$$

Then the equation governing $v$ becomes

$$
\frac{v^{2} J(v)}{\left(v^{2}-v+1\right)^{5 / 4}}=\frac{2 c b^{2}}{9(-a)^{5 / 2}} \exp \left(-\int_{0}^{\tilde{t}} k\left(-\frac{a(\tau)}{b(\tau)}, \tau\right) d \tau\right) .
$$

Now the escape time $T_{0}$ can be determined by

$$
\frac{c b^{2}\left(T_{0}\right)}{(-a)^{5 / 2}\left(T_{0}\right)} \exp \left(-\int_{0}^{\varepsilon T_{0}} k\left(-\frac{a(\tau)}{b(\tau)}, \tau\right) d \tau\right)=\frac{3}{5} .
$$

3.2. Cubic nonlinear oscillator. Consider the following cubic nonlinear oscillator:

$$
\frac{d^{2} y}{d t^{2}}+\varepsilon k_{1}(y, \tilde{t}) \frac{d y}{d t}+a_{1}(\tilde{t}) y+b_{1}(\tilde{t}) y^{3}=0
$$

The potential corresponding to (3.4) is

$$
V\left(y_{0}, a_{1}, b_{1}\right)=\frac{1}{2} a_{1}(\tilde{t}) y_{0}^{2}+\frac{1}{4} b_{1}(\tilde{t}) y_{0}^{4} .
$$

It can be seen from (3.20) that the potential $V$ has a minimum at $y_{0}=0$ for the case of $a_{1}(\tilde{t})>0$ and the system oscillates around the center $y_{r}=0$. For the case of $a_{1}(\tilde{t})<0$ and $b_{1}(\tilde{t})>0$, the potential $V$ has two minimums at $y_{0}= \pm \sqrt{-a_{1}(\tilde{t}) / b_{1}(\tilde{t})}$. The system has two families of oscillations centered about $y_{r}= \pm \sqrt{-a_{1}(\tilde{t}) / b_{1}(\tilde{t})}$, which are moving slowly with time. 
3.2.1. Case of $a_{1}(\tilde{t})>0$ and $b_{1}(\tilde{t})<0$. Similar to the quadratic nonlinear oscillator, we can get approximate solution of leading order

$$
\begin{gathered}
y_{0}=\sqrt{\frac{-2 a_{1} v}{b_{1}(1+v)}} \operatorname{sn}(K \varphi, v), \\
\omega(\tilde{t})=\frac{-c_{1} b_{1}(1+v)}{2 a_{1} v K(v) L(v)} \exp \left(-\int_{0}^{\tilde{t}} k_{1}(0, \tau) d \tau\right),
\end{gathered}
$$

where

$$
L(v)=\int_{0}^{K} c n^{2}(u, v) d n^{2}(u, v) d u=\frac{1}{3 v}[(1+v) E(v)-(1-v) K(v)] .
$$

The equation governing $v$ becomes

$$
\frac{v^{2} L^{2}(v)}{(1+v)^{3}}=\frac{c_{1}^{2} b_{1}^{2}}{4 a_{1}^{3}} \exp \left(-2 \int_{0}^{\tilde{t}} k_{1}(0, \tau) d \tau\right)
$$

where constant $c_{1}$ can be determined by the initial values of the system.

The escape time $T_{0}$ can be solved from (3.24):

$$
\frac{c_{1}^{2} b_{1}^{2}\left(T_{0}\right)}{a_{1}^{3}\left(T_{0}\right)} \exp \left(-2 \int_{0}^{\varepsilon T_{0}} k_{1}(0, \tau) d \tau\right)=\frac{2}{9}
$$

Here, the fact that $L(v) \rightarrow 2 / 3$ as $v \rightarrow 1$ has been used. For more details of this subsection, readers can refer to [15].

3.2.2. Case of $a_{1}(\tilde{t})<0$ and $b_{1}(\tilde{t})>0$. Here we are concerned only with the oscillation around the right-hand side center. Similar to the above sections, we can get the asymptotic solution of leading order

$$
\begin{gathered}
y_{0}=\sqrt{\frac{2 a_{1}}{b_{1}(v-2)}} d n(K \varphi, v), \\
\omega(\tilde{t})=\frac{c_{1} b_{1}(v-2)}{2 a_{1} v^{2} K(v) M(v)} \exp \left(-\int_{0}^{\tilde{t}} k_{1}\left(\sqrt{-\frac{a(\tau)}{b(\tau)}}, \tau\right) d \tau\right),
\end{gathered}
$$

where

$$
M(v)=\int_{0}^{K} s n^{2}(u, v) c n^{2}(u, v) d u=\frac{1}{3 v^{2}}[(2-v) E(v)-2(1-v) K(v)] .
$$

The equation governing $v$ becomes

$$
\frac{v^{4} M^{2}(v)}{(v-2)^{3}}=\frac{c_{1}^{2} b_{1}^{2}}{4 a_{1}^{3}} \exp \left(-2 \int_{0}^{\tilde{t}} k_{1}\left(\sqrt{-\frac{a(\tau)}{b(\tau)}}, \tau\right) d \tau\right)
$$


The escape time $T_{0}$ can be determined by (3.28):

$$
\frac{c_{1}^{2} b_{1}^{2}\left(T_{0}\right)}{a_{1}^{3}\left(T_{0}\right)} \exp \left(-2 \int_{0}^{\varepsilon T_{0}} k_{1}\left(\sqrt{-\frac{a_{1}(\tau)}{b_{1}(\tau)}}, \tau\right) d \tau\right)=-\frac{4}{9} .
$$

Here, the fact that $M(v) \rightarrow 1 / 3$ as $v \rightarrow 1$ has been used.

\section{Examples}

Example 4.1. Consider the following quadratic nonlinear oscillator:

$$
\begin{gathered}
\frac{d^{2} y}{d t^{2}}-\varepsilon\left(\frac{1}{1+\varepsilon t}-y^{3}\right) \frac{d y}{d t}+(1+\varepsilon t)^{2} y-(1+\varepsilon t)^{5 / 2} y^{2}=0 \\
y(0)=0.5, \quad \dot{y}(0)=0 .
\end{gathered}
$$

From initial conditions we can obtain $\varphi_{0}=1, v(0)=0.5$, and $c=0.27312$. The escape time $T_{0}$ can be solved from (3.14):

$$
T_{0}=\frac{1}{\varepsilon}\left(\frac{3}{5 c}-1\right)=\frac{1.197}{\varepsilon} .
$$

Comparisons of asymptotic results from (4.3) and numerical results are shown in Table 4.1. In this paper, numerical results are obtained by software Mathematica.

Example 4.2. Consider the following quadratic nonlinear oscillator:

$$
\begin{gathered}
\frac{d^{2} y}{d t^{2}}-\varepsilon\left(3+\varepsilon t+y-y^{2}\right) \frac{d y}{d t}-(1+\varepsilon t)^{2} y+(1+\varepsilon t) y^{2}=0 \\
y(0)=0.1, \quad \dot{y}(0)=0 .
\end{gathered}
$$

From initial conditions we can obtain $\varphi_{0}=1, v(0)=0.878$, and $c=0.57514$. The escape time $T_{0}$ can be solved from (3.18):

$$
T_{0}=\frac{0.1819}{\varepsilon}
$$

Comparisons of asymptotic results from (4.6) and numerical results are shown in Table 4.2.

Example 4.3. Consider the strongly nonlinear oscillator

$$
\begin{gathered}
\frac{d^{2} y}{d t^{2}}-\varepsilon \frac{1}{1+\varepsilon t} \frac{d y}{d t}+(1+\varepsilon t) \sin y-\frac{2}{\pi}(1+\varepsilon t) y=0, \\
y(0)=\frac{\pi}{3}, \quad \dot{y}(0)=0 .
\end{gathered}
$$

The leading-order equation corresponding to (2.2) has the form

$$
\omega^{2}(\tilde{t}) \frac{\partial^{2} y_{0}}{\partial t^{+2}}+(1+\varepsilon t) \sin y_{0}-\frac{2}{\pi}(1+\varepsilon t) y_{0}=0
$$


Table 4.1. Escape time from potential well of system (4.1).

\begin{tabular}{lccc}
\hline \multicolumn{1}{c}{$\varepsilon$} & Asymptotic result & Numerical result & Error $(\%)$ \\
\hline 0.1 & 11.97 & 14.83 & 19 \\
0.01 & 119.7 & 124.4 & 3.8 \\
0.001 & 1197 & 1236 & 3.2 \\
0.0001 & 11970 & 12301 & 2.7 \\
\hline
\end{tabular}

Its energy integral is

$$
\frac{\omega^{2}(\tilde{t})}{2}\left(\frac{\partial y_{0}}{\partial t^{+}}\right)^{2}+V\left(y_{0}\right)=E_{0}(\tilde{t})
$$

where

$$
V\left(y_{0}\right)=-(1+\varepsilon t) \cos y_{0}-\frac{1}{\pi}(1+\varepsilon t) y_{0}^{2}+1+\varepsilon t
$$

is the potential. With this potential, the integral of (4.10) cannot be expressed in terms of any elemental or known functions. Approximate approaches must be used. The approximate potential method was first proposed in [14] to deal with a strongly nonlinear oscillator resulting from the free-electron laser (FEL), where the potential was approximated by a polynomial of degree three. Note that the potential $V\left(y_{0}\right)$ has a minimum point at $y_{0}=0$ and two maximum points at $y= \pm \pi / 2$. We may seek a polynomial of degree four to approximate it (see [4] for more details). Denoting it by

$$
\bar{V}(y)=\frac{1}{2} a_{1}(\tilde{t}) y^{2}+\frac{1}{4} b_{1}(\tilde{t}) y^{4}
$$

where the coefficients are chosen such that

$$
\bar{V}=V, \quad \bar{V}^{\prime}=0 \quad \text { at } y=0, y=\frac{\pi}{2},
$$

we get

$$
a_{1}(\tilde{t})=\frac{4(4-\pi)}{\pi^{2}}(1+\varepsilon t), \quad b_{1}(\tilde{t})=-\frac{16(4-\pi)}{\pi^{4}}(1+\varepsilon t) .
$$

Substituting $\bar{V}$ for $V$ in (4.10) and integrating it, we can obtain the approximate solution of (3.21) and the escape time of (3.25). From initial conditions, we can obtain $\varphi_{0}=1$, $v(0)=0.285714$, and $c=0.431393$. From (3.25), the approximate escape time $T_{0}$ is

$$
T_{0}=\frac{1.529}{\varepsilon} \text {. }
$$

Comparisons of asymptotic results from (4.15) and numerical results are shown in Table 4.3.

If the potential (4.11) is expanded as Taylor series of fourth order, the escape time corresponding to $(4.15)$ is $\tilde{T}_{0}=1.082 / \varepsilon$, which has an unacceptable error of about $26 \%$. 
Table 4.2. Escape time from potential well of system (4.4).

\begin{tabular}{lccc}
\hline \multicolumn{1}{c}{$\varepsilon$} & Asymptotic result & Numerical result & Error $(\%)$ \\
\hline 0.01 & 18.19 & 18.49 & 1.6 \\
0.001 & 181.9 & 180.4 & 0.8 \\
0.0001 & 1819 & 1779 & 2.3 \\
\hline
\end{tabular}

Table 4.3. Escape time from potential well of system (4.7).

\begin{tabular}{lccc}
\hline \multicolumn{1}{c}{$\varepsilon$} & Asymptotic result & Numerical result & Error $(\%)$ \\
\hline 0.1 & 15.29 & 17.25 & 11 \\
0.01 & 152.9 & 147.5 & 3.7 \\
0.001 & 1529 & 1467 & 4.2 \\
0.0001 & 15290 & 14635 & 4.5 \\
\hline
\end{tabular}

The reason is that Taylor series expansion is valid only for small oscillation, while the approximate potential works for relatively large oscillation.

\section{Conclusions}

(1) The multiple scales method and character of modulus of Jacobian elliptic function are applied to drive the escape time from the potential wells of quadratic and cubic nonlinear oscillators. The method of approximate potential makes the result more accurate than that of Taylor series expansion.

(2) Examples show that the asymptotic results are in good agreement with the numerical results. It should be noted that the amount of computations is about the same though $\varepsilon$ decreases. However, if one uses a numerical method to solve (4.1), (4.4), and (4.7), as $\varepsilon$ decreases these systems become stiff and the computing time increases rapidly and may produce large system errors.

\section{References}

[1] D. L. Bosley and J. Kevorkian, Free-electron laser with very slow wiggler taper, IEEE J. Quantum Electron. 27 (1991), 1078-1089.

[2] J. B. Buchler and H. Einchhorn (eds.), Chaotic Phenomena in Astrophysics, Annals of the New York Academy of Sciences, vol. 497, New York Academy of Sciences, New York, 1987.

[3] P. F. Byrd and M. D. Friedman, Handbook of Elliptic Integrals for Engineers and Scientists, 2nd ed., Die Grundlehren der mathematischen Wissenschaften, vol. 67, Springer, New York, 1971.

[4] J. Cai and Y. P. Li, Approximate potentials with applications to strongly nonlinear oscillators with slowly varying parameters, Shock and Vibration 10 (2003), no. 5-6, 379-386.

[5] R. Chacon, F. Balibrea, and M. A. Lopez, Role of parametric resonance in the inhibition of chaotic escape from a potential well, Phys. Lett. A 235 (1997), 153-158.

[6] W. T. Coffey, D. S. F. Crothers, and S. V. Titov, Escape times for rigid Brownian rotators in a bistable potential from the time evolution of the Green function and the characteristic time of the probability evolution, Phys. A 298 (2001), 330-350. 
[7] M. J Davis and R. E. Wyatt, Surface-of-section analysis in the classical theory of multiphoton absorption, Chem. Phys. Lett. 86 (1982), no. 3, 235-241.

[8] M. Frey and E. Simiu, Phase space transport and control of escape from a potential well, Phys. D 95 (1996), 128-143.

[9] M. R. Frey, A Wiener filter, state-space flux-optimal control against escape from a potential well, IEEE Trans. Automat. Contr. 41 (1996), no. 2, 216-223.

[10] J. Hoffnagle, R. G. DeVoe, L. Reyna, and R. G. Brewer, Order-chaos transition of two trapped ions, Phys. Rev. Lett. 61 (1988), 255-258.

[11] J. Kevorkian and J. D. Cole, Perturbation Methods in Applied Mathematics, Applied Mathematical Sciences, vol. 34, Springer, New York, 1981.

[12] J. Kevorkian and Y. P. Li, Explicit approximations for strictly nonlinear oscillators with slowly varying parameters with applications to free-electron lasers, Stud. Appl. Math. 78 (1988), no. 2, 111-165.

[13] G. E. Kuzmak, Asymptotic solutions of nonlinear second order differential equations with variable coefficients, Prikl. Mat. Meh. 23 (1959), 515-526 (Russian).

[14] Y. P. Li, Free electron lasers with variable parameter wigglers, a strictly nonlinear oscillator with slowly varying parameters, Ph.D. thesis, University of Washington, Washington, 1987.

[15] _ Elapsed time of periodic motion with negative damping, Appl. Math. Mech. (English Ed.) 13 (1992), no. 8, 719-723.

[16] T. Poston and I. N. Stewart, Catastrophe Theory and Its Applications, Surveys and Reference Works in Mathematics, no. 2, Pitman, London, 1978.

[17] R. Tchoukuegno, B. R. N. Nbendjo, and P. Woafo, Linear feedback and parametric controls of vibrations and chaotic escape in a $\Phi^{6}$ potential, Internat. J. Non-Linear Mech. 38 (2003), no. $4,531-541$.

Jianping Cai: Department of Mathematics, Zhangzhou Teachers College, Fujian 363000, China E-mail address: mathcai@hotmail.com

Y. P. Li: Department of Mathematics, Zhongshan University, Guangzhou 510275, China E-mail address: ypli@umac.mo

Xiaofeng Wu: Department of Mathematics, Zhongshan University, Guangzhou 510275, China E-mail address:wuxiaof@21cn.com 


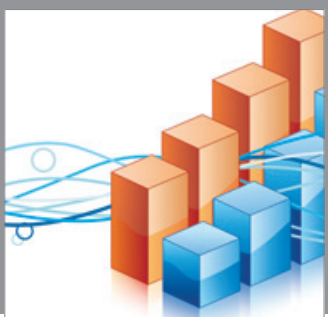

Advances in

Operations Research

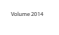

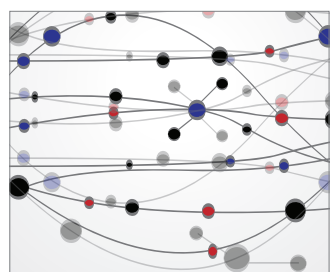

\section{The Scientific} World Journal
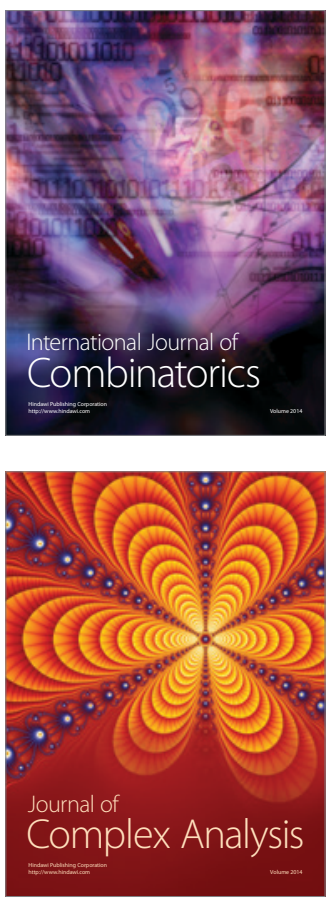

International Journal of

Mathematics and

Mathematical

Sciences
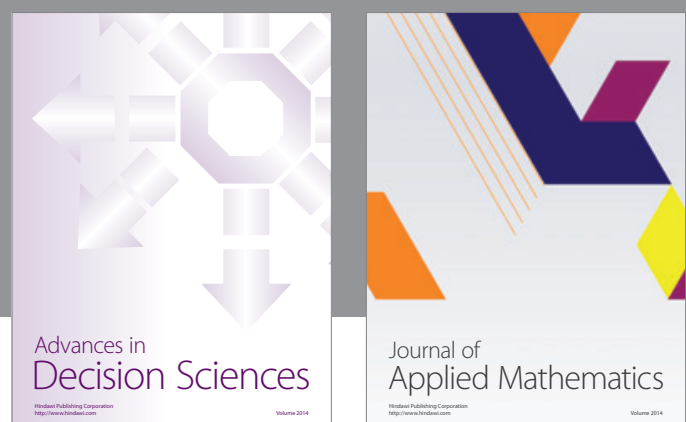

Journal of

Applied Mathematics
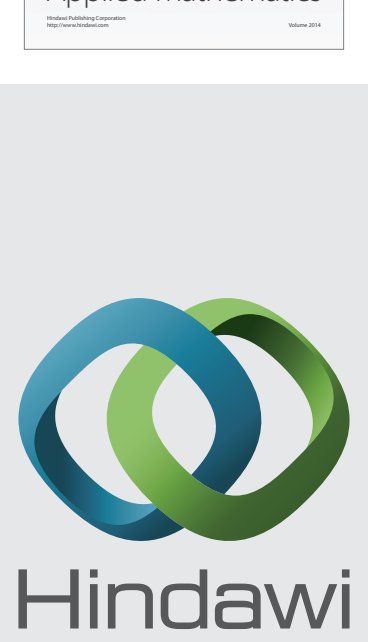

Submit your manuscripts at http://www.hindawi.com
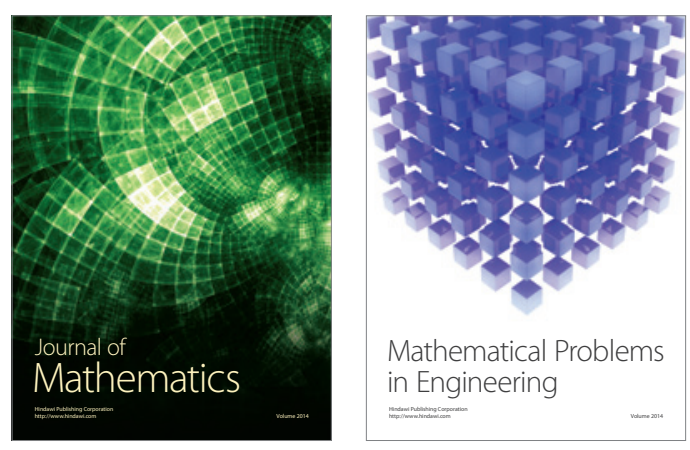

Mathematical Problems in Engineering
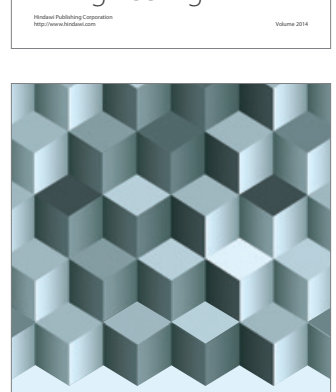

Journal of

Function Spaces
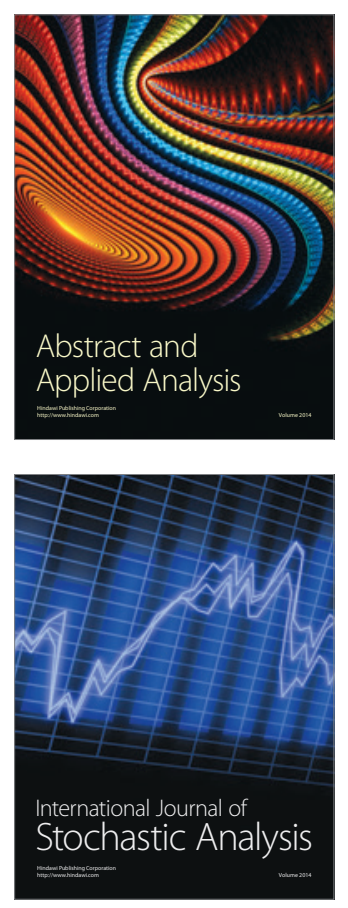

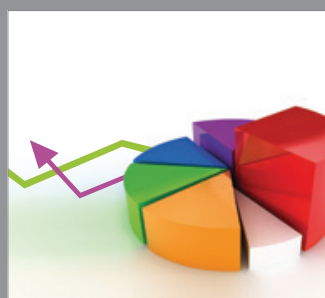

ournal of

Probability and Statistics

Promensencen
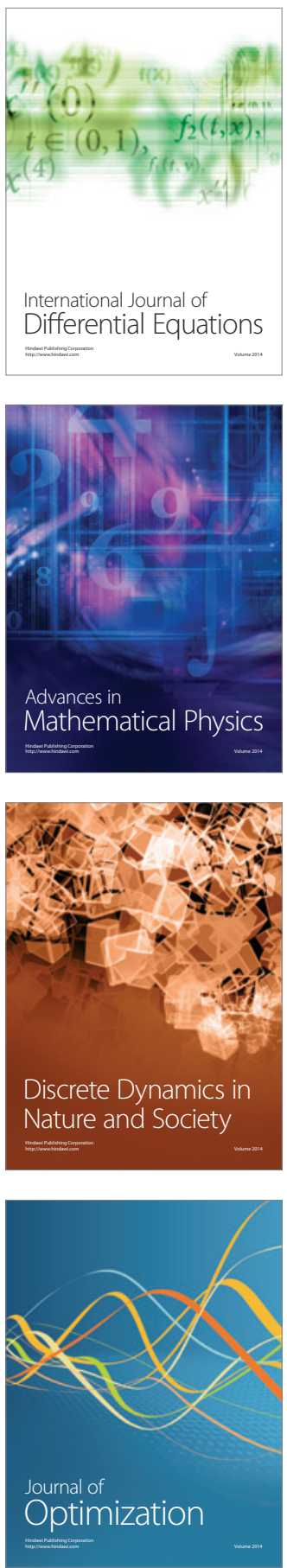